\title{
¿HERENCIA O CONTAGIO? POSTURAS SOBRE LA TRANSMISIÓN DE LA TUBERCULOSIS EN COSTA RICA
}

Inheritance or contagion? Postures on the transmission of tuberculosis in Costa Rica

\author{
Monserrat Rojas Madrigal \\ Universidad de Costa Rica, Costa Rica \\ monroma.86@gmail.com
}

Recibido: $13-01-2019$

Aprobado: 29-03-2019

\begin{abstract}
Monserrat Rojas Madrigal es licenciada en Antropología con énfasis en Arqueología de la Universidad de Costa Rica. Se desempeña como investigadora Independiente.
\end{abstract}

\section{RESUMEN}

Se muestra la controversia suscitada por las autoridades médicas durante el año 1934, acerca de la forma de transmisión de la tuberculosis. Para esto, se analizan las diferentes posturas de algunos médicos interesados en el tema durante esa época, los cuales, con base a sus experiencias laborales con pacientes enfermos de tisis, escriben en la revista médica de Costa Rica u opinan tanto en la asamblea médica como en el segundo congreso médico celebrado en el país durante ese mismo año, acerca de las formas de contagio de la tuberculosis en la población costarricense.

Palabras clave: tuberculosis; medicina; controvérsia; herencia; contagio

\section{ABSTRACT}

It shows the controversy raised by the medical authorities during the year 1934, about the way of transmission of tuberculosis. For this, the different positions of some physicians interested in the subject during that time are analyzed, which, based on their work experiences with patients suffering from phthisis, write in the medical journal of Costa Rica or opinions both in the medical assembly and in the second medical congress celebrated in the country during that same year, about the forms of contagion of tuberculosis in the Costa Rican population.

Keywords: tuberculosis; medicine; controversy; inheritance; contagion 


\section{Introducción}

Con motivo de la asamblea médica celebrada en el país en el Sanatorio Carlos Durán el día 8 de abril de 1934, se desencadena una discusión que se extiende en varios números de la revista médica de Costa Rica sobre las formas de contagio de la enfermedad, en donde llegaron a participar los médicos Ricardo Jiménez Núñez, Werner Rotter, Mario Luján Fernández, Antonio Peña Chavarría, Julio Ovares Arias, el científico Clodomiro Picado Twight así como el director de la revista Joaquín Zeledón Alvarado. Dicha discusión se continuó también durante el segundo congreso médico centroamericano realizado durante este mismo año, donde participaron los médicos antes mencionados y otras luminarias nacionales y extranjeras de la época.

Bajo esta coyuntura, se pretende explorar las posturas de los médicos antes mencionados con respecto a la transmisión de la tuberculosis en el país, con el fin de comprender las ideas que estaban detrás del trabajo desempeñado en la lucha contra dicha enfermedad. Por lo que a nivel metodológico se empleará el método analítico-descriptivo de tres números de la Revista Médica de Costa Rica de 1934 y las Memorias del segundo Congreso Médico Centroamericano (1934), tratando de darles la palabra a los protagonistas de dichas controversias.

\section{II. ¿Herencia o contagio?}

Durante la asamblea médica sobre tuberculosis llevada a cabo en el Sanatorio Durán en 1934, el Dr. Peña Chavarría se refirió a la tuberculosis en los niños y niñas, aseverando que los y las mismas se curaban usualmente mediante la acción climática y dietética. Sin embargo, indica:

El campesino nuestro...tiene poca alergia tuberculosa [por lo tanto] ante todo [se debe] proteger al campesino que tiene una alergia evidente y por ende llega a ser víctima imprescindible del contagio tuberculoso (Zeledón, marzo-abril 1934, p. 146)

Demuestra, a su vez, con porcentajes obtenidos al realizar la prueba de Mantoux (conocida también como tuberculina) en niños de 0 a 4 años, que en citadinos la incidencia de tuberculosis se presentaba más alta que en el campo, teniendo San José un $17 \%$ positivas mientras que en el campo $8 \%$ positivas; pero al realizar 58 autopsias tipo tuberculosis infantil solo 9 respondían a citadinos, mientras que el resto eran casos provenientes del campo. 
Es decir, en este momento el Dr. Peña Chavarría, observa una incidencia mayor en el desarrollo de la enfermedad en infantes del campo en contraposición con los casos de la ciudad, con lo cual se considera que los primeros tienen una mayor predisposición a la "alergia tuberculosa". Sobre esto el Dr. J. Ovares confirma dichas hipótesis haciendo referencia al caso de Italia, diciendo lo siguiente:

La afluencia de las gentes de campo a la ciudad ofrece ese peligro innegable de contagio, desprovistos como están de inmunidad adquirida. Mussolini que ha podido comprender hasta la intimidad de los grandes problemas sanitarios de Italia, prohibió la invasión de las ciudades por los elementos rurales, como resultado la tuberculosis ha disminuido en Italia (Zeledón, marzo-abril 1934, p. 146).

Ahora bien, el Dr. M Luján, haciendo un análisis de su experiencia como médico del Patronato Nacional de la Infancia (PANI), señala que un elemento a tomar en cuenta en la población infantil contagiada es la pobreza y la miseria, señalando como un ambiente propicio para la tuberculosis (Zeledón, marzo-abril 1934). Hasta este punto, parece que existía la visión del campesinado como personas más sensibles al contagio de la tuberculosis, problema que se extiende a la población infantil. Se exaltan las políticas sanitarias de Mussolini, al prohibir el éxodo del campo a la ciudad, razón por la cual se vio una disminución de peste blanca en Italia. Y, por último, se menciona brevemente la recurrencia de condiciones de pobreza en los infantes con tuberculosis atendidos por el médico del PANI.

Posteriormente, el Dr. Werner Rotter, director del Servicio de Patología del Hospital San Juan de Dios, publica los estudios realizados sobre 342 casos a los que le realizó autopsias entre agosto de 1930 y noviembre de 1933, comparándolo a su vez con un artículo de la revista "Die Tuberkulose", en donde se analizó un material parecido en Europa. Sus principales impresiones indican que al menos durante la primera década de vida de una persona contagiada la mortalidad solía ser más alta, es decir, solían presentarse en Costa Rica una mayor mortalidad en la población infantil con edades inferiores a los 10 años, mientras que en Europa las cifras eran menores. De igual forma, encontró una amplia incidencia en muertes por tuberculosis en población entre los 20 y los 50 años, aunque menor que los casos Europeos (Rotter, julio-agosto 1934). 
Según la experiencia del Dr. Rotter, los pacientes de tuberculosis contraen la enfermedad por la aspiración de bacilos y la fuente de aspiración es la expectoración de personas con dicha enfermedad; razón por la cual el foco primario de infección se encontraba generalmente en los pulmones (Rotter, julio-agosto 1934).

El director de la revista médica de Costa Rica Joaquín Zeledón, se afianza del estudio del Dr. Rotter, para incitar mayor acción del estado en la lucha antituberculosa exaltando la necesidad de que el gobierno

comprenda ese dogma de Higiene Pública, sin el cual toda campaña va al fracaso: Autonomía técnica, organización casi militar y DINERO [sic] (Zeledón, julio-agosto 1934, p. 219).

Para Zeledón el problema de la tuberculosis descansaba sobre una base social, en donde consideraba que la desocupación, la ignorancia, la pobreza, la mala alimentación, los problemas con las viviendas, los vicios, las malas condiciones sanitarias, la mortalidad elevada y la tuberculosis debían ser consideradas como enfermedades sociales; debido a esto abogaba por la medicina social, en donde la prevención se centrara como un pilar.

Durante el segundo congreso médico centroamericano, se plantean varias ideas sobre el desarrollo de la tuberculosis en la población costarricense, así como el control de la enfermedad en contraposición a otros países latinoamericanos.

Se compara la mortalidad por tuberculosis con países americanos o Europeos que tenían "una cultura sanitaria superior", aunado a las bondades del clima en donde se asentaba el grueso de la población lejos de los

rigores tropicales de las zonas costeñas y por otros factores epidemiológicos influyentes, entre ellos el racial, pues la mayoría de la población del país es blanca (el 80 \% según censo de 1921) (República de Costa Rica, 1935, p. 257).

A esto se adjunta una nota al pie sobre dicho censo en donde la población estaba compuesta por blancos $80.2 \%$, mestizos $14.1 \%$, negros $4.1 \%$, indios $0.9 \%$, mulatos $0.5 \%$, amarillos $0.1 \%$ y otros no clasificados $0.1 \%$. A lo largo del congreso se deja entrever, la concepción que la población blanca de cierta forma tiene mayores cualidades físicas para combatir la enfermedad; por ejemplo, se indica que: 
el curso especial que sigue la tuberculosis en adultos de razas que no han logrado una relativa inmunización explica el por qué en Costa Rica, los escasos indígenas de tribus aisladas que se trasladan a residir en las ciudades, son víctimas frecuentes de tuberculosis que evolucionan rápidamente (República de Costa Rica, 1935, p.260).

Se muestra además que existía una diferenciación por sexo en cuanto a la incidencia de la enfermedad, de modo tal que las mujeres eran afectadas usualmente entre los 15 y los 30 años, mientras que los hombres presentaban un ascenso en el índice de mortalidad rondando los 40 años. Este fenómeno se explicaba relacionándolo con la pubertad, en donde se consideraba que llegaba de manera más tardía en hombres que en mujeres (República de Costa Rica, 1935).

El último número de la Revista Médica de Costa Rica de1934 dedica dos artículos al tema de la propagación de la tuberculosis. El primero de ellos escrito por el Dr. Jiménez Núñez, en donde escribe una breve reseña en cuanto a las discusiones sobre las formas de transmisión de la enfermedad, concluyendo que los médicos mostraban tres posturas:

La mayoría de los sabios es contagionista, una minoría se aferra a la herencia y otro grupo más prudente ha contestado: "Si el contagio de la tuberculosis es un punto innegable, el papel de la herencia en su transmisión no debe ser negado, contrariamente a lo que los tratados nos enseñan" (Jiménez, diciembre 1934, p. 257).

A partir de aquí el Dr. Jiménez Núñez presenta su postura en cuanto a la forma de transmisión de la enfermedad aduciendo

la predisposición hereditaria, es decir la herencia de terreno: el hijo de tuberculosos vendrá a la vida en condiciones predispuestas para la adquisición de la enfermedad, justificando la aparición, en una familia, de casos sucesivos y repetidos de tuberculosis (Jiménez, diciembre 1934).

Este médico respalda su postura citando casos tales como el personal hospitalario que al estar expuesto a los esputos de los pacientes no se enfermaba, explicando dicho hecho en relación a una ascendencia indemne, es decir, que la familia de dicho personal nunca hubiese padecido de tuberculosis. Este hecho lo relaciona además con que en un matrimonio que estuviese padeciendo esta enfermedad era más peligroso para la descendencia que para los progenitores, exponiendo que "en el medio familiar la contaminación del niño es la regla; la del cónyuge la excepción" (Jiménez, diciembre 1934, p. 259). 
Indica además que un hombre adulto sin antecedentes hereditarios de tuberculosis, puede convivir con tísicos sin enfermarse; afirmando que el contagio exige condiciones muy especiales, admitiendo entonces que personas que proceden de

regiones incivilizadas en las cuales no existe el alcoholismo ni la tisis, y que de pronto cambian de medio y pasan a vivir entre tísicos, pueden adquirir la enfermedad (Jiménez, diciembre 1934, p.259).

Para finalizar, a criterio del Dr. Jiménez Núñez la tuberculosis se hallaba en retroceso, aunque discrepa en la postura de los contagionistas que consideran que las medidas de desinfección y aislamiento han sido las causantes, argumentando que:

estas medidas solo afectan a un número ínfimo de enfermos, a los de los sanatorios; a cada paso encontramos tísicos que ignoran su enfermedad y esparcen a su alrededor 72,000 millones de bacilos diariamente. Por dicha el riesgo de contaminación por el esputo del tísico parece ser muy escaso, si es que existe (Jiménez, diciembre 1934, p. 262).

Para este médico son otras las razones que explican el descenso en los casos de tuberculosis:

En primer lugar, el más importante, es la inmunidad adquirida por los sobrevivientes después de que la selección natural ha eliminado a los individuos de menor resistencia, inmunidad que se transmite por herencia. En segundo lugar, el descubrimiento precoz de la enfermedad que la pone en condiciones admirables para curarla con los métodos terapéuticos modernos que nos brindan los sanatorios: colapsoterapia, curas de reposo, etc. La enfermedad se extinguirá como se ha extinguido la lepra, pero no por el aislamiento de los tísicos sino cuando haya la suficiente energía para oponerse a la creación de nuevas generaciones de tuberculosos no permitiendo la unión de sujetos indemnes con bacilares (Jiménez, diciembre 1934, p. 262).

El Dr. Zeledón Alvarado (diciembre, 1934), retoma los argumentos planteados por el Dr. Jiménez Núñez, presentando la discusión suscitada en 1933 entre Auguste Lumiére y Albert Calmette, el primero defendiendo la herencia como forma de contagio de la tuberculosis, mientras que el segundo sosteniendo el contagio por esputo; y basándose en dicha controversia entrevista al Dr. Picado Twight, acerca de la polémica escrita por dichos autores y replicada por médicos de todo el mundo, incluida Costa Rica, como se ha presentado a lo largo de este artículo. De esta forma el Dr. Picado Twight señala "me parece una discusión estéril ya que desde el punto de vista biológico, el problema está perfectamente mal planteado" (Zeledón, diciembre 1934, p. 275), en tanto según este científico 
la herencia debía ser una cualidad positiva dentro de la especie, por lo que al hablar de la herencia solo podría hablarse de la resistencia a la infección, puesto que "El tener la tuberculosis o ser suceptible [sic] a ella es justamente el carácter negativo inherente a todo el género humano" (Zeledón, diciembre 1934, p. 276). El Dr. Picado plantea además que la solución al problema deriva en tres cosas

Mucha higiene, mucha comida, y como atravesamos una etapa de transición entre el período intacto y el "premunizado" por la existencia de tuberculosis frustras, mucha TUBERCULOSIS [sic] (Zeledón, diciembre 1934, p. 277).

\section{Conclusiones}

Desde los primeros escritos sobre la tuberculosis existió una discusión sobre las características que la conformaban, preocupando principalmente su forma de contagio. Esta discusión se mantuvo inclusive después de descubierto el bacilo causante de la misma, formándose "bandos" de científicos y médicos, los cuales recolectaban evidencia dentro de su experiencia con los enfermos de tisis para hacer sus conjeturas. De esta forma se consolidó el grupo de los contagionistas, quedando relegados aquellos que pensaban en la herencia como la forma de transmisión de la tuberculosis.

La postura que estaba relacionada con la herencia tenía una larga trayectoria, por lo que aun después del descubrimiento del bacilo de koch (causante de la enfermedad), la instauración de políticas públicas en el país para la contención de la enfermedad y la aparente reducción en el número de casos, no se logró convencer a aquellos que asumían a la herencia como raíz de este mal.

Existió una tercera postura, un poco más cautelosa a la hora de abordar la comprensión sobre el contagio de la tuberculosis, posicionándose en un punto medio entre las perspectivas antes expuestas.

Si bien no se ahonda en el tema de políticas públicas en este artículo, hay que reconocer que existía un gran interés de parte de la comunidad médica en el tema de la tuberculosis, su tratamiento y su contención, razón por la cual, la definición de la forma de contagio de dicha enfermedad es de gran interés, suscitando tan apasionadas respuestas de parte de los médicos. 
Claramente, la tuberculosis tuvo un amplio impacto social hasta la implementación de la vacuna BCG, no solamente en Costa Rica, sino también en el resto del mundo; siendo una enfermedad confusa y engañosa para los científicos y médicos, por lo que aún con los adelantos de la época, se les dificultaba hacer una interpretación precisa de la misma.

\section{BIBLIOGRAFÍA}

Jiménez, R. (diciembre 1934). Como se propaga la tuberculosis. Revista Médica de Costa Rica 2 (11), 257-262.

República de Costa Rica. (1935). Memorias del segundo congreso médico centroamericano. San José: Imprenta Nacional.

Rotter, W. (julio-agosto 1934). Observaciones anatomo-patológicas sobre la tuberculosis en Costa Rica. Revista Médica de Costa Rica 1(9), 193-203.

Zeledón, J. (marzo-abril 1934). Asamblea Médica sobre Tuberculosis. Revista Médica de Costa Rica 1 (6-7), 142-147.

Zeledón, J. (julio-agosto 1934). Se agita el problema de la tuberculosis en Costa Rica. Revista Médica de Costa Rica 1(9), 219-221.

Zeledón, J. (diciembre 1934). Herencia y tuberculosis. Revista Médica de Costa Rica 2 (11), 273-277. 